\title{
Fragilidade em idosos que vivem na comunidade: prevalência e fatores associados
}

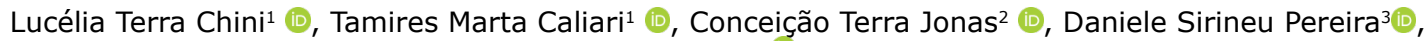 \\ Jair Lício Ferreira Santos ${ }^{4}$, Altacílio Aparecido Nunes ${ }^{4}$
}

\begin{abstract}
RESUMO
Fundamentos: Idosos que vivem na comunidade são propensos a desenvolver fragilidade, considerada como um estado clinicamente identificável que aumenta a vulnerabilidade a resultados adversos e prediz incapacidade e mortalidade na população idosa. Objetivo: Identificar a prevalência e fatores associados à fragilidade em idosos que vivem em uma comunidade. Método: Trata-se de um inquérito domiciliar transversal e analítico, de abordagem quantitativa realizado com 854 idosos que vivem na comunidade. A fragilidade foi mensurada pela Edmonton Frail Scale (EFS). A associação entre fragilidade e variáveis sociodemográficas e de condições clínicas foi mensurada pela análise múltipla por regressão logística. Resultados: A prevalência de fragilidade encontrada neste estudo foi de 12,3\% (IC95\%: 10,1 a 14,5). O modelo de regressão logística mostrou que as variáveis estatisticamente associadas à fragilidade foram: queda recorrente, uso de dispositivo para auxílio à marcha, polifarmácia, autopercepção ruim de saúde, dependência nas atividades básicas e instrumentais de vida diária. Conclusão: A prevalência de fragilidade em idosos foi baixa em comparação a outros estudos nacionais que empregaram a EFS. Os resultados indicaram múltiplos fatores associados à fragilidade modificáveis. Assim, a investigação da síndrome da fragilidade bem como dos seus fatores relacionados passíveis de prevenção são ações a serem incluídas na prática clínica.
\end{abstract}

Palavras-chave: Idoso, Idoso fragilizado, Prevalência, Vulnerabilidade em saúde, Atenção primária à saúde.

1 Universidade Federal de Alfenas. Escola de Enfermagem, (MG) Brasil.

2 Prefeitura Municipal de Alfenas, (MG) Brasil.

3 Universidade Federal de Minas Gerais. Escola de Fisioterapia e Terapia Ocupacional

4 Universidade de São Paulo. Faculdade de Medicina de Ribeirão Preto, (SP), Brasil 


\section{INTRODUÇÃO}

O processo de envelhecimento da população mundial envolve alterações fisiológicas, as quais associadas aos fatores sociodemográficos (sexo, estado civil, etnia), fatores socioeconômicos (escolaridade, renda), estilo de vida, eventos da vida, meio ambiente e fatores genéticos podem ocasionar o surgimento de síndromes geriátricas, dentre elas, a fragilidade ${ }^{1}$.

A fragilidade em idosos tornou-se uma condição de grande preocupação das sociedades e seus sistemas de saúde no que diz respeito aos esforços destinados a aumentar a expectativa de vida saudável e os cuidados de saúde na população idosa ${ }^{2-3}$. A expressão fragilidade tem sido constantemente utilizada pelos profissionais de saúde para caracterizar idosos mais fracos e vulneráveis ${ }^{4}$.

Ainda não há uma definição de fragilidade universalmente aceita. Entretanto, evidencia-se um recente entendimento da fragilidade como uma síndrome complexa que envolve cinco componentes: vulnerabilidade, gênese (fatores de risco), características (comportamento do sistema complexo), fenótipo (físico, nutricional, cognitivo, psicológico e social) e resultados adversos relacionados à saúde ${ }^{4}$.

Considerando a inexistência de um padrãoouro aceito globalmente sobre a definição de fragilidade, o conceito mais utilizado foi proposto por Fried e colaboradores ${ }^{5}$, que operacionalizaram a fragilidade como tendo três ou mais dos cinco critérios: perda de peso não intencional, exaustão autorreferida, fraqueza, baixa velocidade de caminhada e baixa atividade física.

Assim, a fragilidade consiste em uma condição clinicamente identificável de maior vulnerabilidade a resultados desfavoráveis devido à imperfeita resolução da homeostase após um evento estressante 6 . Essa condição clínica tem consequências negativas sobre o processo de envelhecimento, como incapacidade funcional, aumento da utilização de cuidados de saúde e morte prematura, levando ao aumento dos custos e impondo desafios aos gestores do sistema de saúde?.

A prevalência da fragilidade aumenta juntamente com o rápido crescimento da população idosa ${ }^{8}$. Entretanto, a prevalência de fragilidade varia entre os estudos a depender da definição de fragilidade adotada. Uma revisão sistemática evidenciou que a prevalência de fragilidade entre idosos residentes na comunidade com idade $\geq 65$ anos variou de $4,0 \%$ a $59,1 \%{ }^{9}$.

Os idosos que vivem na comunidade são propensos a desenvolver fragilidade ${ }^{10}$. Os fatores de risco para fragilidade incluem fatores sociodemográficos como idade, sexo, estado civil, nível de escolaridade e fatores físicos como composição corporal e limitação de mobilidade ${ }^{11-14}$. No entanto, uma revisão sistemática de estudos longitudinais identificou uma gama mais ampla de fatores de risco e proteção, incluindo fatores biológicos, de estilo de vida e psicológicos ${ }^{15}$.

Embora constitua uma condição de desenvolvimento gradual, a síndrome da fragilidade é passível de prevenção e reabilitação, o que representa grandes possibilidades para profissionais de saúde ${ }^{6}$. Com um crescente interesse mundial no envelhecimento saudável, o conhecimento dos fatores que predispõem a fragilidade em idosos é fundamental para subsidiar o desenho de intervenções preventivas e a elaboração de políticas públicas adequadas, visando a redução de sua incidência e consequências deletérias de forma a manter a capacidade funcional e a qualidade de vida na velhice. Diante desse contexto, este estudo tem por objetivo identificar a prevalência e fatores associados à fragilidade em idosos que vivem em uma comunidade.

\section{MATERIAIS E MÉTODO}

Trata-se de um estudo transversal e analítico, de abordagem quantitativa realizado em um município do Sul de Minas Gerais, o qual, de acordo com o último censo, possuía uma população de 9.113 idosos $^{16}$.

A população do estudo foi definida com base no universo de idosos cadastrados nas 14 unidades de Estratégia Saúde da Família (ESF) da zona urbana de Alfenas-MG. Definiram-se como campo de estudo quatro unidades de ESF pelo fato de possuir o maior número de idosos, um total de 1825 sujeitos, e por ser campo de prática e de ensino de maior proximidade da pesquisadora principal. Inicialmente, determinou-se que a amostra deste estudo seria composta por 1000 pessoas idosas selecionadas por um processo de amostragem aleatória estratificada com partilha proporcional. Utilizou-se um adicional 
de $10 \%$ para perdas e recusas. O número de idosos retirado de cada estrato foi de 334 na ESF 1 (total de 555 idosos), 293 na ESF 2 (total de 486 idosos), 255 na ESF 3 (total de 423 idosos) e de 218 na ESF 4 (total de 362 idosos). Dos 1100 idosos sorteados, 28 não aceitaram participar da pesquisa, 33 faleceram, 46 mudaram de endereço e 139 apresentaram um dos critérios de exclusão. Considerando a obtenção de uma amostra representativa, a dificuldade de recrutar pesquisadores de campo e ao término do prazo estimado para coleta de dados não foram realizados novos sorteios. Dessa forma, a amostra final foi composta por 854 participantes.

Foram incluídos no estudo idosos com idade igual ou superior a 60 anos, conscientes, orientados, capazes de interagir durante a entrevista e de se locomover mesmo que utilizasse algum dispositivo de auxílio para marcha (andador, bengala ou muleta). Foram excluídos da amostra idosos com alterações cognitivas detectáveis pelo Mini Exame do Estado Mental -MEEM (o ponto de corte foi ajustado de acordo com o nível educacional) ${ }^{17}$, com doença infecciosa em fase aguda, com fraturas nos membros inferiores, realização de artroplastia de quadril e joelho nos últimos três meses, com hipoacusia severa e perda total da visão.

A entrevista com os participantes foi realizada por pesquisadores de campo no domicílio do idoso. Estes pesquisadores foram devidamente treinados e calibrados ao longo da realização do estudo.

A variável dependente foi mensurada pela Edmonton Frail Scale (EFS) ${ }^{18}$, uma escala de avaliação de fragilidade em idosos, elaborada em 2009 por pesquisadores da Universidade de Alberta, Edmonton, Canadá e foi traduzida e adaptada para o contexto brasileiro em $2009^{19}$. Tal escala avalia nove domínios: cognição, estado geral de saúde, independência funcional, suporte social, uso de medicamentos, nutrição, humor, continência e desempenho funcional, investigados por 11 itens. Sua pontuação máxima é 17 e representa o nível mais elevado de fragilidade. Os escores para análise da fragilidade são: 0-4, não apresenta fragilidade; 5-6, aparentemente vulnerável; 7-8, fragilidade leve; 9-10, fragilidade moderada; 11 ou mais, fragilidade severa. Para análise dos dados no presente estudo, os resultados da variável dependente foram categorizados em dois níveis: sem fragilidade (pontuação de 0 a 6 ) e com fragilidade (maior e igual a 7 pontos) $)^{20}$.
As variáveis independentes estudadas foram as relacionadas às condições sociodemográficas e às condições clínicas de saúde: faixa etária (60 -79 anos e $\geq 80$ anos), sexo (feminino, masculino), mora sozinho (não/sim), escolaridade ( 0 a 4 anos e 5 e mais anos), queda nos últimos 12 meses ( $\operatorname{sim} /$ não), queda recorrente (presença de duas ou mais quedas nos últimos 12 meses - sim/ não), fratura de quadril ou pernas nos últimos 12 meses ( $\operatorname{sim} /$ não), uso de dispositivo para auxílio à marcha (bengala, muleta, andador - sim/não), polifarmácia (uso de 4 ou mais medicamentos, exceto vitaminas e fitoterápicos - sim/não), psicotrópicos (medicamentos pertencentes às classes farmacêuticas dos antiepiléticos, antiparkinsonianos, antipsicóticos, ansiolíticos, hipnóticos e sedativos, antidepressivos, psicoestimulantes, psicolépticos, psicoanalépticos e fármacos antidemência conforme o sistema de classificação Anatomical Therapeutical Classification - ATC (sim/não) ${ }^{21}$.

Ainda sobre as variáveis independentes, a autopercepção de saúde foi avaliada por meio da pergunta: "De modo geral, como o(a) Sr(a) descreveria sua saúde: excelente, muito boa, boa, razoável ou ruim?22. As respostas excelentes, muito boa, boa foram recategorizadas como "boa" e as respostas razoável e ruim como "ruim"22.

A realização das Atividades Básicas de Vida Diária (ABVD) foi avaliada através da Escala de Katz (versão atualizada pelo Hartforf Institute for Geriatric Nursing), que contém seis itens com duas opções de respostas cada (1 ponto para independência e 0 para dependência) ${ }^{23}$. Foram considerados dependentes os idosos que pontuaram de 0 a 5 pontos e independentes aqueles que alcançaram 6 pontos $^{24}$.

As Atividades Instrumentais de Vida Diária (AIVD) foram avaliadas mediante aplicação da versão brasileira da Escala de Lawton e Brody ${ }^{25}$. Tal escala contém sete itens e cada item apresenta três tipos de resposta: independência (3), dependência parcial (2), dependência total (1), com pontuação máxima de 21 pontos. As respostas foram recategorizadas em dependente ( 7 a 20 pontos) e independente ( 21 pontos) para realização de AIVD25.

A realização de Atividades Avançadas de Vida Diária (AAVD) ${ }^{26}$ foi avaliada através de 12 questões envolvendo os domínios social, produtivo e físico/lazer: a) contato com outras pessoas por cartas, telefone ou e-mail; b) visita a amigos e familiares em suas casas; c) cuidado ou assistência a outras pessoas (incluindo 
cuidado pessoal, transporte, compras para familiares ou amigos); d) trabalho voluntário fora de casa; e) viagem para fora da cidade pernoitando pelo menos uma noite; f) participação em algum programa de exercícios regulares (esportes, exercícios físicos, caminhadas e grupos de práticas corporais); g) convida pessoas para virem à sua casa para refeições ou lazer; h) sai com outras pessoas para lugares públicos como restaurante ou cinema; i) realização de alguma atividade manual, artesanato ou atividade artística; j) participação em atividades sociais organizadas (clubes, grupos comunitários ou religiosos, centros de convivência de idosos, bingo); I) faz uso de computador, incluindo a Internet; $\mathrm{m}$ ) dirige veículos a motor. As questões foram respondidas considerando duas opções de resposta: $\operatorname{sim}(0)$ e não (1). Assim, idosos que pontuaram de 0 a 5 pontos foram considerados independentes e idosos que pontuaram 6 ou mais pontos foram classificados como dependentes ${ }^{26}$.

Para avaliar a presença de sintomas depressivos foi utilizada a forma abreviada da Escala de Depressão Geriátrica (EDG) ${ }^{27}$, a qual contém 15 itens. Considerou-se a presença de sintomas depressivos uma pontuação $\geq 6$ pontos na EDG ${ }^{27}$.

O medo de cair foi avaliado através da versão brasileira da Falls Efficacy Scale - International $(\text { FES-I })^{28}$. Tal instrumento apresenta 16 itens com quatro possibilidades de respostas, com respectivos escores de um a quatro. O escore total pode variar de 16 a 64 . Considerou-se baixo risco potencial de quedas uma pontuação de 16 a 22 pontos e alto risco potencial de quedas uma pontuação de 23 ou mais pontos na FES-I ${ }^{29}$.

A análise estatística compreendeu o cálculo da análise bivariada seguida de análise múltipla por regressão logística. A análise bivariada foi feita empregando-se o teste qui-quadrado $\left(X^{2}\right)$, a razão de prevalência (RP) e seu respectivo intervalo de confiança a $95 \%$ para investigar a existência de associações entre as variáveis independentes e a fragilidade. Para a análise final, utilizou-se a Regressão Logística, sendo incluídas no modelo as variáveis independentes que estiveram mais fortemente associadas com a fragilidade na análise bivariada (até o nível de significância $<0,20$ ). Para a análise final, considerou-se um nível de significância de $5 \%(p<0,05)$. Os dados foram analisados no software estatístico Med Calc versão 16.4.1.

O presente estudo foi aprovado pelo Comitê de Ética em Pesquisa (CEP) do Hospital das Clínicas da Faculdade de Medicina de Ribeirão Preto (Parecer 1.575.252) e todos os participantes assinaram ou carimbaram com digital do polegar direito o Termo de Consentimento Livre e Esclarecido e receberam uma cópia do mesmo.

\section{RESULTADOS}

Participaram do estudo 854 idosos com idade igual ou superior a 60 anos. Com relação às características sociodemográficas, observou-se que a faixa etária predominante foi entre 60 e 79 anos, o que representou $82,7 \%$ da amostra em estudo, com média de idade de $71,87$ anos ( $\mathrm{DP}=7,62)$. A maioria das pessoas idosas era do sexo feminino $(494 ; 57,8 \%)$, brancas $(642 ; 75,1 \%)$ e casadas (523; 61,2\%). Ademais, $757(88,6 \%)$ idosos moravam com seus familiares, $663(77,6 \%)$ referiram possuir até quatro anos de estudo, 734 (85,9\%) eram aposentadas e 341 (39,9\%) possuíam renda familiar de um salário mínimo.

A prevalência de fragilidade encontrada neste estudo foi de $12,3 \%$, sendo maior para o sexo feminino (70,5\%) e para faixa etária entre 60 e 79 anos (61,9\%).

Outras características do grupo revelaram que 263 idosos $(30,7 \%)$ caíram nos últimos 12 meses, $99(11,5 \%)$ caíram de forma recorrente nos últimos 12 meses e $11(3,6 \%)$ tiveram fraturas de quadril ou pernas nos últimos 12 meses. O uso de DAM, polifarmácia e psicotrópicos foi apontado por 51 (5,9\%), 388 (45,4\%) e 208 (24,3\%) idosos, respectivamente. Com relação à autopercepção de saúde, 298 (34,8\%) consideram sua saúde ruim. Quanto à capacidade funcional, 82 (9,6\%) idosos são dependentes para atividades de vida diária básicas, $582(68,1 \%)$ são dependentes para atividades instrumentais e $384(44,9 \%)$ são dependentes para atividades avançadas. O rastreio positivo para depressão foi identificado em 6,5\% (56) dos idosos. Com relação ao medo de cair, 34,3\% (293) revelaram alto risco potencial de quedas. Os resultados da análise bivariada são apresentados na Tabela 1 .

As variáveis que, após analise múltipla por regressão logística, se mantiveram estatisticamente associadas à fragilidade foram: queda recorrente, uso de DAM, polifarmácia, autopercepção ruim de saúde, dependência nas atividades básicas e instrumentais de vida diária (Tabela 2). 
Tabela 1. Resultado da análise bivariada entre fragilidade e variáveis independentes em idosos da comunidade ( $n=854)$.

\begin{tabular}{|c|c|c|c|c|c|c|c|}
\hline \multirow[t]{3}{*}{ Variáveis independentes } & \multicolumn{4}{|c|}{ Fragilidade } & \multirow{3}{*}{$\mathbf{R P *}$} & \multirow{3}{*}{ IC $95 \%+$} & \multirow{3}{*}{ p-valor } \\
\hline & \multicolumn{2}{|c|}{ Não } & \multicolumn{2}{|c|}{ Sim } & & & \\
\hline & $\mathbf{n}$ & $\%$ & $\mathbf{n}$ & $\%$ & & & \\
\hline \multicolumn{8}{|l|}{ Faixa etária } \\
\hline $60-79$ anos & 642 & 85,7 & 65 & 61,9 & 1 & & \\
\hline 80 anos e mais & 107 & 14,3 & 40 & 38,1 & 2,95 & $2,08-4,20$ & $<0,0001$ \\
\hline \multicolumn{8}{|l|}{ Sexo } \\
\hline Masculino & 329 & 43,9 & 31 & 29,5 & 1 & & \\
\hline Feminino & 420 & 56,1 & 74 & 70,5 & 1,74 & $1,16-2,58$ & 0,0062 \\
\hline \multicolumn{8}{|l|}{ Mora sozinho } \\
\hline Não & 668 & 89,2 & 89 & 84,8 & 1 & & \\
\hline Sim & 81 & 10,8 & 16 & 15,2 & 1,40 & $0,86-2,28$ & 0,1742 \\
\hline \multicolumn{8}{|l|}{ Escolaridade } \\
\hline 5 e mais anos & 183 & 24,4 & 8 & 7,6 & 1 & & \\
\hline 0 a 4 anos & 566 & 75,6 & 97 & 92,4 & 3,49 & $1,72-7,05$ & 0,0005 \\
\hline \multicolumn{8}{|l|}{ Queda } \\
\hline Não & 544 & 72,6 & 47 & 44,8 & 1 & & \\
\hline Sim & 205 & 27,4 & 58 & 55,2 & 2,77 & $1,94-3,95$ & $<0,0001$ \\
\hline \multicolumn{8}{|l|}{ Queda recorrente } \\
\hline Não & 687 & 91,7 & 68 & 64,8 & 1 & & \\
\hline Sim & 62 & 8,3 & 37 & 35,2 & 4,14 & $2,95-5,83$ & $<0,0001$ \\
\hline \multicolumn{8}{|l|}{ Fratura } \\
\hline Não & 728 & 97,2 & 95 & 90,5 & 1 & & \\
\hline Sim & 21 & 2,8 & 10 & 9,5 & 2,79 & $1,62-4,81$ & 0,0002 \\
\hline \multicolumn{8}{|l|}{ Uso de DAM } \\
\hline Não & 723 & 96,5 & 80 & 76,2 & 1 & & \\
\hline Sim & 26 & 3,5 & 25 & 23,8 & 4,92 & $3,47-6,97$ & $<0,0001$ \\
\hline \multicolumn{8}{|l|}{ Polifarmácia } \\
\hline Não & 440 & 58,7 & 26 & 24,8 & 1 & & \\
\hline Sim & 309 & 41,3 & 79 & 75,2 & 3,65 & $2,39-5,56$ & $<0,0001$ \\
\hline \multicolumn{8}{|l|}{ Psicotrópicos } \\
\hline Boa & 580 & 77,4 & 66 & 62,9 & 1 & & \\
\hline $\operatorname{Sim}$ & 169 & 22,6 & 39 & 37,1 & 1,83 & $1,27-2,64$ & 0,0011 \\
\hline \multicolumn{8}{|l|}{ Autopercepção saúde } \\
\hline Good & 528 & 70,5 & 28 & 26,7 & 1 & & \\
\hline Ruim & 221 & 29,5 & 77 & 73,3 & 5,13 & $3,40-7,72$ & $<0,0001$ \\
\hline \multicolumn{8}{|l|}{ Atividades Básicas de Vida Diária } \\
\hline Independente & 696 & 90,2 & 76 & 9,8 & 1 & & \\
\hline Dependente & 53 & 64,6 & 29 & 35,4 & 3,59 & $2,50-5,16$ & $<0,0001$ \\
\hline \multicolumn{8}{|c|}{ Atividades Instrumentais de Vida Diária } \\
\hline Independente & 192 & 25,6 & 80 & 76,2 & 1 & & \\
\hline Dependente & 557 & 74,4 & 25 & 23,8 & 6,84 & $4,47-10,47$ & $<0,0001$ \\
\hline Atividades Avançadas de Vida Di & & & & & & & \\
\hline Independente & 386 & 51,5 & 84 & 80,0 & 1 & & \\
\hline Dependente & 363 & 48,5 & 21 & 20,0 & 0,30 & $0,19-0,48$ & $<0,0001$ \\
\hline Sintomas depressivos - GDS & & & & & & & \\
\hline Rastreio negativo para depressão & 715 & 95,5 & 83 & 79,0 & 1 & & \\
\hline Rastreio positivo para depressão & 34 & 4,5 & 22 & 21,0 & 3,77 & $2,57-5,54$ & $<0,0001$ \\
\hline Medo de cair & & & & & & & \\
\hline Baixo risco potencial de quedas & 521 & 69,7 & 39 & 37,1 & 1 & & \\
\hline Alto risco potencial de quedas & 227 & 30,3 & 66 & 62,9 & 3,23 & $2,23-4,68$ & $<0,0001$ \\
\hline
\end{tabular}

Fonte: dos autores. *RP - Razão de prevalência; †IC - Intervalo de Confiança; DAM - Dispositivo de Auxílio à Marcha. 
Tabela 2. Fatores associados à fragilidade em idosos da comunidade $(n=854)$.

\begin{tabular}{|c|c|c|c|}
\hline Variáveis independentes & $\mathbf{R P} *$ & IC95\%† & p-valor \\
\hline \multicolumn{4}{|l|}{ Queda recorrente } \\
\hline Não & 1 & & \\
\hline Yes & 3,15 & $1,44-6,89$ & 0,0039 \\
\hline \multicolumn{4}{|l|}{ Uso de DAM } \\
\hline Não & 1 & & \\
\hline Sim & 2,64 & $1,27-5,49$ & 0,0092 \\
\hline \multicolumn{4}{|l|}{ Polifarmácia } \\
\hline Não & 1 & & \\
\hline Sim & 2,59 & $1,41-4,74$ & 0,0020 \\
\hline \multicolumn{4}{|l|}{ Autopercepção saúde } \\
\hline Boa & 1 & & \\
\hline Ruim & 6,11 & $3,42-10,89$ & $<0,0001$ \\
\hline \multicolumn{4}{|l|}{$\begin{array}{l}\text { Atividades Básicas de } \\
\text { Vida Diária }\end{array}$} \\
\hline \multicolumn{4}{|l|}{ Independente } \\
\hline Dependente & 2,87 & $1,42-5,80$ & 0,0033 \\
\hline \multicolumn{4}{|l|}{$\begin{array}{l}\text { Atividades } \\
\text { Instrumentais de Vida } \\
\text { Diária }\end{array}$} \\
\hline Independente & 1 & & \\
\hline Dependente & 4,83 & $2,61-8,92$ & $<0,0001$ \\
\hline
\end{tabular}

Source: from the authors. * PR - Prevalence ratio; † Cl - Confidence Interval, DAM - Walking Aid Device.

\section{DISCUSSÃO}

$\mathrm{Na}$ amostra de idosos da comunidade do Município de Alfenas, a prevalência de fragilidade foi de $12,3 \%$, diferente da encontrada em outros estudos nacionais que utilizaram a mesma ferramenta para identificar a fragilidade. Em estudo transversal com 339 idosos (60 anos ou mais) residentes em Juiz de Fora, MG, a prevalência de fragilidade encontrada foi de $35,7 \%{ }^{20}$. Na pesquisa conduzida em Montes Claros, MG, com idosos da comunidade, a prevalência de fragilidade foi de $41,3 \%^{30}$. Outros estudos nacionais evidenciaram prevalência semelhante a do presente estudo, porém, utilizaram os componentes do Fenótipo de Fragilidade de Fried para mensurar o desfecho ${ }^{31-32}$. No sentido de comparar os resultados obtidos a partir da aplicação da Edmonton Frail Scale (EFS) e do Fenótipo de Fragilidade de Fried em uma mesma amostra, um estudo realizado na Colômbia encontrou prevalências semelhantes $(8,9 \%$ e $7,9 \%$ respectivamente) $)^{33}$.
No cenário mundial, a prevalência de fragilidade em idosos da comunidade, em média, é de $10,7 \%$, variando de $4,0 \%$ a $59,1 \%{ }^{9}$. Uma recente revisão sistemática e meta-análise sobre prevalência de fragilidade em idosos da comunidade que vivem em países de baixa e média renda, mostrou que esses dados variam de 3,9\% (China) a 51,4\% (Cuba) ${ }^{34}$. Esse mesmo estudo identificou que a prevalência de fragilidade no Brasil varia de $7,7 \%$ a $41,3 \%{ }^{34}$. Cabe destacar que essas discordâncias em relação à prevalência se devem às diferentes ferramentas empregadas para mensurar a fragilidade e também às características de cada população estudada.

A condição de fragilidade implica em maior vulnerabilidade a eventos estressores, com consequências deletérias à saúde do idoso. A identificação de fatores associados à fragilidade torna-se, então, relevante para direcionamento de ações para manutenção da independência e funcionalidade da pessoa idosa.

Dentre os fatores investigados, a ocorrência de quedas recorrentes apresentou associação com a condição de fragilidade. Outras pesquisas indicam 
associação entre quedas recorrentes e fragilidade ${ }^{35-38}$. Os resultados de uma meta-análise revelaram que idosos frágeis demonstraram maior risco de queda e de quedas recorrentes quando comparados com idosos robustos ${ }^{39}$. Idosos frágeis tem uma diminuição da capacidade de reserva funcional, além de, geralmente, apresentarem maior número de doenças crônicas e reações adversas a medicamentos. Em conjunto, tal cenário pode implicar em maiores alterações e déficits na manutenção do equilíbrio e coordenação, predispondo ao aumento do risco de quedas ${ }^{40}$.

No presente estudo, a fragilidade também se mostrou associada ao uso de dispositivo para auxílio à marcha (DAM), assim como outros estudos ${ }^{20,41-42}$. Estudos apontam que DAM são indicadores de déficits do controle postural e de risco de quedas ${ }^{43-44}$. Desse modo, idosos que utilizam tais dispositivos apresentam mais problemas de mobilidade do que aqueles que não usam ${ }^{45}$, além de apresentarem maior risco de cair e sofrer uma lesão ${ }^{46}$. Todavia, não foram encontrados estudos que investigassem de modo específico as relações entre o uso de DAM e a fragilidade.

A fragilidade permaneceu associada à polifarmácia, corroborando com outros estudos ${ }^{24,47}$. A polifarmácia em idosos está associada a vários indicadores negativos de saúde, como comprometimento funcional, fraturas, quedas, hospitalização e mortalidade ${ }^{48-50}$. Assim, a polifarmácia é considerada fator de risco para fragilidade em pessoas idosas, uma vez que a sobreposição de vários medicamentos e seus efeitos adversos podem exacerbar essa condição ${ }^{24,51}$.

A associação entre fragilidade e autopercepção negativa de saúde observada neste estudo também foi registrada em outras pesquisas ${ }^{24,52-53}$. A fragilidade é caracterizada pela redução da capacidade para responder às condições de estresse e maior susceptibilidade a eventos adversos, podendo levar ao agravamento de doenças e ao ciclo crescente de debilidade ${ }^{24,52}$, o que pode explicar a associação entre fragilidade e autopercepção negativa de saúde.

A síndrome da fragilidade foi fortemente relacionada à dependência tanto em atividades instrumentais quanto atividades básicas de vida diária ${ }^{30,54-57}$. Na literatura essa relação foi identificada tanto em estudos transversais quanto longitudinais, demonstrando a necessidade da abordagem precoce por parte dos profissionais da saúde para evitar o declínio funcional da pessoa idosa. Um inquérito domiciliar transversal realizado no Brasil com 1.609 idosos da comunidade, demonstrou associação com a incapacidade funcional para atividades básicas e instrumentais da vida diária ${ }^{32}$. Estudo transversal com 339 idosos da comunidade de Juiz de Fora, MG, que também utilizou a EFS, evidenciou associação de fragilidade e dependência funcional para realização das atividades instrumentais de vida diária ${ }^{20}$. Um estudo de coorte prospectivo de 10 anos comprovou que idosos frágeis apresentaram duas vezes mais probabilidade de relatar incapacidade funcional ao longo do tempo em comparação com idosos não frágeis ${ }^{58}$.

O emprego da EFS mostrou-se útil para identificação e gerenciamento da fragilidade em pessoas idosas que vivem na comunidade. Ademais, configura-se como uma ferramenta de baixo custo, de fácil e rápida aplicação e que não exige maiores recursos para sua execução, podendo ser aplicada amplamente nas instituições de saúde, em especial, no contexto da atenção primária à saúde.

Este estudo apresenta como limitação o tempo de coleta de dados, o que impossibilitou a realização de novo sorteio para aquisição de novos participantes. Contudo, este trabalho utilizou instrumento validado culturalmente no contexto brasileiro para identificação da síndrome da fragilidade e possui amostra representativa para o emprego dos modelos de regressão logística. Outrossim, o controle de qualidade foi empregado nas etapas do estudo, como treinamento e calibração dos entrevistadores, testagem dos instrumentos, assegurando maior confiabilidade aos dados analisados.

\section{CONCLUSÃO}

A prevalência de fragilidade em idosos evidenciada neste estudo foi de $12,3 \%$, ou seja, baixa em comparação a outros estudos nacionais que empregaram a EFS. Entretanto, foi semelhante a outros estudos nacionais que utilizaram os componentes do Fenótipo de Fragilidade de Fried para mensurar o desfecho. A fragilidade esteve associada à queda recorrente, uso de dispositivo para auxílio à marcha, polifarmácia, autopercepção ruim de saúde, dependência nas atividades básicas e instrumentais de vida diária.

Ressalta-se, que a identificação da prevalência da fragilidade em pessoas idosas e o entendimento dos seus fatores associados, são fundamentais para 
o planejamento e implementação de ações de saúde destinadas a essa população, no sentido de prevenir, retroceder ou evitar a progressão desta síndrome.

\section{REFERÊNCIAS}

1. Gobbens RJ, Luijkx KG, Wijnen-Sponselee MT, Schols JM. Towards an integral conceptual model of frailty. J Nutr Health Aging. 2010;14(3):175-81. DOI: https:// doi.org/10.1007/s12603-010-0045-6

2. Clegg A, Young J, Iliffe S, Rikkert MO, Rockwood K. Frailty in elderly people. Lancet. 2013; 381(9868):752-62. DOI: https://doi.org/10.1016/S0140-6736(12)62167$\underline{9}$

3. Bandeen-Roche $\mathrm{K}$, Seplaki CL, Huang J, Buta B, Kalyani RR, Varadhan R, et al. Frailty in older adults: a nationally representative profile in the United States. Journals Gerontol Ser A Biol Sci Med Sci. 2015;70(11):1427-34. DOI: https://doi.org/10.1093/ gerona/glv133

4. Junius-Walker $U$, Onder $G$, Soleymani D, Wiese B, Albaina $O$, Bernabei $R$, et al. The essence of frailty: a systematic review and qualitative synthesis on frailty concepts and definitions. Eur J Intern Med. 2018;56:3-10. DOI: https://doi.org/10.1016/j.ejim.2018.04.023

5. Fried LP, Tangen C, Walston J, Newman AB, Hirsch C, Gottdiener J, et al. Frailty in Older Adults: evidence for a phenotype. J Gerontol. 2001;56(3):M146-57. DOI: https://doi.org/10.1093/gerona/56.3.M146

6. Morley JE, Vellas B, Kan GA van, Anker SD, Bauer JM, Bernabei $R$, et al. Frailty conensus: a call to action. J Am Med Dir Assocation. 2013;14(6):392-7. DOI: https://doi.org/10.1016/j.jamda.2013.03.022

7. Chen X, Mao G, Leng SX. Frailty syndrome: an overview. Clin Interv Aging. 2014;9:433-41. DOI: https://doi. org/10.2147/CIA.S45300

8. Hoogendijk EO, Afilalo J, Ensrud KE, Kowal P, Onder G, Fried LP. Frailty: implications for clinical practice and public health. Lancet. 2019;394(10206):1365-1375. DOI: https://doi.org/10.1016/S0140-6736(19)31786$\underline{6}$

9. Collard RM, Boter H, Schoevers RA, Oude Voshaar RC. Prevalence of frailty in community-dwelling older persons: a systematic review. J Am Geriatr Soc. 2012;60(8):1487-92. DOI: https://doi.org/10.1111/ j.1532-5415.2012.04054.x

10. Ofori-Asenso R, Chin KL, Mazidi M, Zomer E, Ilomaki J, Zullo AR, et al. Global incidence of frailty and prefrailty among community-dwelling older adults: a systematic review and meta-analysis. JAMA Netw Open. 2019; 2(8):e198398. DOI: https://doi.org/10.1001/ jamanetworkopen.2019.8398

11. Jung $H$, Kim M, Lee $Y$, Won CW. Prevalence of physical frailty and its multidimensional risk factors in korean community-dwelling older adults: findings from korean frailty and aging cohort study. Int $\mathrm{J}$ Environ Res Public Health. 2020;17(21):7883. DOI: https://doi. org/10.3390/ijerph17217883

12. Poli S, Cella A, Puntoni M, Musacchio C, Pomata $M$, Torriglia $D$, et al. Frailty is associated with socioeconomic and lifestyle factors in communitydwelling older subjects. Aging Clin. Exp. Res. 2017; 29:721-728. DOI: https://doi.org/10.1007/s40520$\underline{016-0623-5}$

13. Vaingankar JA, Chong SA, Abdin E, Picco L, Chua BY, Shafie $S$, et al. Prevalence of frailty and its association with sociodemographic and clinical characteristics, and resource utilization in a population of Singaporean older adults. Geriatr. Gerontol. Int. 2017; 17:14441454. DOI: https://doi.org/10.1111/ggi.12891

14. Wong $\mathrm{CH}$, Weiss $\mathrm{D}$, Sourial $\mathrm{N}$, Karunananthan $\mathrm{S}$, Quail JM, Wolfson C, et al. Frailty and its association with disability and comorbidity in a community-dwelling sample of seniors in Montreal: A cross-sectional study. Aging Clin. Exp. Res. 2010; 22:54-62. DOI: https://doi.org/10.1007/BF03324816

15. Feng $Z$, Lugtenberg $M$, Franse $C$, Fang $X$, Hu S, Jin C, et al. Risk factors and protective factors associated with incident or increase of frailty among communitydwelling older adults: a systematic review of longitudinal studies. PLoS One. 2017;12(6):e0178383. DOI: https://doi.org/10.1371/journal.pone.0178383.

16. Instituto Brasileiro de Geografia e Estatística. Cidades [Internet]. Brasília: Ministério do Planejamento, Orçamento e Gestão [cited 2019 Ago 20]; Disponível em: http://cidades.ibge.gov.br/painel/historico. php? lang $=\&$ codmun $=310160 \&$ search $=$ minas gerais\%7Calfenas.

17. Bertolucci PH, Brucki SM, Campacci SR, Juliano Y. O Mini-Exame do Estado Mental em uma população geral: impacto da escolaridade. Arq Neuropsiquiatr. 1994;52(1):1-7. DOI: http://dx.doi.org/10.1590/ S0004-282X1994000100001

18. Rolfson DB, Majumdar SR, Tsuyuki RT, Tahir A, Rockwood K. Validity and reliability of the Edmonton Frail Scale. Age Ageing. 2006;35(5):526-9. DOI: https://doi.org/10.1093/ageing/afl041

19. Fabrício-Wehbe SCC, Schiaveto FV, Vendrusculo TRP, Haas VJ, Dants RAS, Rodrigues RAP. Cross-cultural adaptation and validity of the "Edmonton Frail Scale - EFS" in a brazilian elderly sample. Rev Lat Am Enfermagem. 2009;17(6):1-7. DOI: https://doi. org/10.1590/S0104-11692009000600018

20. Cruz DT, Vieira MT, Bastos RR, Leite ICG. Fatores associados à fragilidade em uma população de idosos da comunidade. Rev. Saúde Pública. 2017; 51:106. DOI: http://dx.doi.org/10.11606/s15188787.2017051007098

21. World Health Organization. Anatomical Therapeutic Chemical (ATC) [Internet]. Oslo: Collaborating Centre for Drug Statistics Methodology [cited 2019 Ago 10]; Available from: https://www.whocc.no/. 
22. Ribeiro EG, Matozinhos FP, Guimarães GL, Couto AM, Azevedo RS, Mendoza IYQ. Autopercepção de saúde e vulnerabilidade clínico-funcional de idosos de Belo Horizonte/Minas Gerais. Rev. Bras. Enferm. 2018; 71(Suppl 2):860-867. DOI: https://doi. org/10.1590/0034-7167-2017-0135

23. Duarte $\mathrm{YAO}$, Andrade $\mathrm{CL}$, Lebrão $\mathrm{ML}$. O index de katz na avaliação da funcionalidade dos idosos. Rev Esc Enferm USP. 2007;41(2):317-25. DOI: https://doi. org/10.1590/S0080-62342007000200021

24. Pegorari MS, Tavares DMS. Factors associated with the frailty syndrome in elderly individuals living in the urban area. Rev Lat Am Enfermagem. 2014;22(5):874-82. DOI: https://doi.org/10.1590/0104-1169.0213.2493

25. Santos RL, Júnior JSV. Confiabilidade da versão brasileira da escala de atividades instrumentais da vida diária. Rev Bras em Promoção da Saúde. 2008;21(4):290-6. Disponível em: http://www. redalyc.org/articulo.oa?id $=40811508010$

26. Dias EG, Andrade FB, Duarte YAO, Santos JLF, Lebrão $M L$. Advanced activities of daily living and incidence of cognitive decline in the elderly: the SABE Study. Cad Saúde Pública. 2015;31(8):1623-35. DOI: https://doi. org/10.1590/0102-311X00125014

27. Almeida OP, Almeida SA. Reliability of the Brazilian version of the geriatric depression scale (GDS) short form. Arq Neuropsiquiatr. 1999;57(2 B):421-6. DOI: https://doi.org/10.1590/S0004-282X1999000300013

28. Camargos FFO, Dias RC, Dias JMD, Freire MTF. Crosscultural adaptation and evaluation of the psychometric properties of the Falls Efficacy Scale - International Among Elderly Brazilians (FES-I-BRAZIL). Rev Bras Fisioter. 2010;14(3):237-43. DOI: https://doi. org/10.1590/S1413-35552010000300010

29. Delbaere K, Close J, Mikolaizak A, Sachdev P, Brodaty $\mathrm{H}$, SR, Lord. The Falls Efficacy Scale International (FES-I). A comprehensive longitudinal validation study. Age Ageing. 2010;39(2):210-6. DOI: https://doi. org/10.1093/ageing/afp225

30. Carneiro JA, Ramos GCF, Barbosa ATF, Medeiros SM, Lima $C$ de $A$, Costa FM, et al. Prevalencia y factores asociados a la fragilidad en ancianos no institucionalizados. Rev Bras Enferm. 2016;69(3):435-42. DOI: https://doi. org/10.1590/0034-7167.2016690304i

31. Moreira V, Lourenco R. Prevalence and factors associated with frailty in an older population from the city of Rio de Janeiro, Brazil: the FIBRA-RJ Study. Clinics. 2013;68(7):979-85. DOI: https://doi. org/10.6061/clinics/2013(07)15

32. Tavares DMS, Corrêa TAF, Dias FA, Ferreira PCS, Pegorari MS. Frailty synrome and socioeconomic and health characteristics among older adults. Colomb Med. 2017;48(3):126-31. DOI: https://doi.org/10.25100/ cm.v48i3.1978

33. Ramírez JUR, Sanabria MOC, Ochoa ME. Aplicación de la Escala de Fragilidad de Edmonton en población colombiana: comparación con los criterios de Fried. Rev
Esp Geriatr Gerontol. 2017;52(6):322-5. DOI: https:// doi.org/10.1016/j.regg.2017.04.001

34. Siriwardhana DD, Hardoon S, Rait G, Weerasinghe MC, Walters KR. Prevalence of frailty and prefrailty among community-dwelling older adults in low-income and middle-income countries: a systematic review and meta-analysis. BMJ Open. 2018;8(3):1-17. DOI: https://doi.org/10.1136/bmjopen-2017-018195

35. Tom SE, Adachi JD, Anderson FA, Boonen S, Chapurlat $\mathrm{RD}$, Compston JE, et al. Frailty and fracture, disability, and falls: A multiple country study from the global longitudinal study of osteoporosis in women. J Am Geriatr Soc. 2013;61(3):327-34. DOI: https://doi. org/10.1111/jgs.12146

36. Wu TY, Chie WC, Yang RS, Kuo KL, Wong WK, Liaw CK. Risk factors for single and recurrent falls: a prospective study of falls in community dwelling seniors without cognitive impairment. Prev Med. 2013;57(5):511-7. DOI: https://doi.org/10.1016/j.ypmed.2013.07.012

37. Vries OJ, Peeters GMEE, Lips P, Deeg DJH. Does frailty predict increased risk of falls and fractures? A prospective population-based study. Osteoporos Int. 2013;24(9):2397-403. DOI: https://doi.org/10.1007/ s00198-013-2303-z

38. Samper-Ternent R, Karmarkar A, Graham J, Reistetter $\mathrm{T}$, Ottenbacher K. Frailty as a predictor of falls in older Mexican Americans. J Aging Health. 2012;24(4):64153. DOI: https://doi.org/10.1177/0898264311428490

39. Cheng $\mathrm{MH}$, Chang SF. Frailty as a risk factor for Falls Among Community Dwelling People: Evidence From a Meta-Analysis. J Nurs Scholarsh. 2017;49(5):529-36. DOI: https://doi.org/10.1111/jnu.12322

40. Kojima G. Frailty as a predictor of future falls among community-dwelling older people: a systematic review and meta-analysis. JAMDA. 2015;16(12):1027-33. DOI: https://doi.org/10.1016/j.jamda.2015.06.018

41. Gale CR, Cooper C, Sayer AA. Prevalence of frailty and disability: findings from the english longitudinal study of ageing. Age Ageing. 2015;44(1):162-5. DOI: https://doi.org/10.1093/ageing/afu148

42. Vieira RA, Guerra RO, Giacomin KC, Vasconcelos KSS, Andrade ACS, Pereira LSM, et al. Prevalência de fragilidade e fatores associados em idosos comunitários de Belo Horizonte, Minas Gerais, Brasil: dados do estudo FIBRA. Cad Saude Publica. 2013;29(8):1631-43. DOI: https://doi.org/10.1590/0102-311X00126312

43. Mahoney J, Sager M, Jalaluddin M. Use of an ambulation assistive device predicts functional decline associated with hospitalization. J Gerontol A Biol Sci Med Sci. 1999;54(2):M83-8. DOI: https://doi.org/10.1093/ gerona/54.2.M83

44. Wright $\mathrm{D}$, Kemp T. The dual-task methodology and assessing the attentional demands of ambulation with walking devices. Phys Ther. 1992;72(4):306-12. DOI: https://doi.org/10.1093/ptj/72.4.306 
45. Jansen S, Schoe J, Rijn MV, Abu-Hanna A, Charante EPMV, Velde NV, et al. Factors associated with recognition and prioritization for falling, and the effect on fall incidence in community dwelling older adults. BMC Geriatr. 2015;15(1):169-79. DOI: https://doi. org/10.1186/s12877-015-0165-2

46. Andersen DA, Roos BA, Stanziano DC, Gonzalez NM, Signorile JF. Walker use, but not falls, is associated with lower physical functioning and health of residents in an assisted-living environment. Clin Interv Aging. 2007;2(1):123-37. DOI: https://doi.org/10.2147/ ciia.2007.2.1.123

47. Gnjidic D, Hilmer SN, Blyth FM, Naganathan V, Cumming RG, Handelsman DJ, et al. High-risk prescribing and incidence of frailty among older community-dwelling men. Clin Pharmacol Ther. 2012;91(3):521-8. DOI: https://doi.org/10.1038/clpt.2011.258

48. Fried TR, O'Leary J, Towle V, Goldstein MK, Trentalange M, Martin DK. Health outcomes associated with polypharmacy in community-dwelling older adults: a systematic review. J Am Geriatr Soc. 2014;62(12):226172. DOI: https://doi.org/10.1111/jgs.13153

49. Lim LM, McStea M, Chung WW, Azmi NN, Aziz SAA, Alwi $S$, et al. Prevalence, risk factors and health outcomes associated with polypharmacy among urban community-dwelling older adults in multiethnic Malaysia. PLoS One. 2017;12(3):1-18. DOI: https:// doi.org/10.1371/journal.pone.0173466

50. Cabello AJP, Gavilán EP, Jiménez FJG, Rodríguez CM, Castillo JDL, Cañas EP. Drug-related mortality among inpatients: a retrospective observational study. Eur J Clin Pharmacol. Eur J Clin Pharmacol. 2016;72(6):7316. DOI: https://doi.org/10.1007/s00228-016-2026-0

51. Gnjidic D, Hilmer SN, Blyth FM, Naganathan V, Cumming RG, Handelsman DJ. High-risk prescribing and incidence of frailty among older communitydwelling men. Clin Pharmacol Ther. 2012;91(3):521-8. DOI: https://doi.org/10.1038/clpt.2011.258
52. Medeiros SM, Silva LSR, Carneiro JA, Ramos GCF, Barbosa ATF, Caldeira AP. Fatores associados à autopercepção negativa da saúde entre idosos não institucionalizados de Montes Claros, Brasil. Ciên Saúde Coletiva. 2016;21(11):3377-86. DOI: https://doi. org/10.1590/1413-812320152111.18752015

53. Rockwood K, Howlett SE, Macknight C, Beattie BL, Bergman $\mathrm{H}$, Hogan DB, et al. Prevalence, attributes, and outcomes of fitness and frailty in communitydwelling older adults: report from the canadian study of health and aging. J Gerontol A Biol Sci Med Sci. 2004;59(12):1310-7. DOI: https://doi.org/10.1093/ gerona/59.12.1310

54. Fhon JRS, Rodrigues RAP, Santos JLF, Diniz MA, Santos EB dos, Almeida VC, et al. Factors associated with frailty in older adults: a longitudinal study. Rev Saude Publica. 2018;52(74):1-8. DOI: https://doi. org/10.11606/s1518-8787.2018052000497

55. Fhon JRS, Diniz MA, Leonardo KC, Kusumota L, Haas VJ, Rodrigues RAP. Frailty syndrome related to disability in the elderly. Acta Paul Enferm. 2012;25(4):589-94. DOI: https://doi.org/10.1590/ S0103-21002012005000016

56. Perna S, Francis MDA, Bologna C, Moncaglieri F, Riva $A$, Morazzoni $P$, et al. Performance of Edmonton Frail Scale on frailty assessment: its association with multidimensional geriatric conditions assessed with specific screening tools. BMC Geriatr. 2017;17(2):1-8. DOI: https://doi.org/10.1186/s12877-016-0382-3

57. Kojima G, Kendrick D, Skelton DA, Morris RW, Gawler S, Iliffe $S$. Frailty predicts short-term incidence of future falls among British community- dwelling older people : a prospective cohort study nested within a randomised controlled trial. BMC Geriatr. 2015;15(155):1-8. DOI: https://doi.org/10.1186/s12877-015-0152-7

58. Snih S Al, Graham JE, Ray LA, Samper- R, Markides $\mathrm{KS}$, Ottenbacher KJ. Frailty and incidence of activities of daily living. J Rehabil Med. 2009;41(11):892-7. DOI: https://doi.org/10.2340/16501977-0424 


\section{Agradecimentos}

À Fundação de Amparo à Pesquisa do Estado de São Paulo (FAPESP) pelo apoio financeiro..

Autor Correspondente:

Lucélia Terra Chini

lu.lucelia@yahoo.com.br

Editor:

Prof. Dr. Felipe Villela Gomes

Recebido: 26/10/2020

Aprovado: 08/03/2021 